\title{
Genesis of the Heaviest Elements in the Milky Way Galaxy
}

\author{
Christopher Sneden, ${ }^{1 *}$ John J. Cowan, ${ }^{1,2}$ \\ ${ }^{1}$ Department of Astronomy \& McDonald Observatory, \\ University of Texas, Austin TX 78712, USA \\ ${ }^{2}$ Department of Physics and Astronomy, \\ University of Oklahoma, Norman, OK 73019, USA
}

*To whom correspondence should be addressed; E-mail: chris@ verdi.as.utexas.edu.

We review the origin and evolution of the heavy elements, those with atomic numbers greater than 30, in the early history of the Milky Way. There is a large star-to-star bulk scatter in the concentrations of heavy elements with respect to the lighter metals, suggesting an early, chemically unmixed and inhomogeneous Galaxy. The relative abundance patterns among the heavy elements are often very different from the Solar System mix, revealing the characteristics of the first element donors in the Galaxy. Abundance comparisons among several halo stars show that the heaviest neutron-capture elements (including Ba and heavier) are consistent with a scaled Solar System rapid neutron-capture abundance distribution, while the lighter such elements do not conform to the Solar pattern. The stellar abundances indicate an increasing contribution from slow neutron-capture process at higher metallicities in the Galaxy. The detection of thorium in halo and globular cluster stars offers a promising, 


\section{independent age-dating technique that can put lower limits on the age of the Galaxy.}

Most beginning chemistry students struggling with the complexities and underlying structure of the Periodic Table will simply accept the existence of the approximately 90 stable elements. Rarely does it occur to them that somewhere and in some way, all of the elements had to be synthesized. Such element generation or nucleosynthesis, through transmutation of one element into another, is a crucial byproduct of stellar energy generation. It has occurred since the birth of the first stars in the Galaxy, and without it life on Earth would not be possible. Here we review the observations and interpretations of heavy elements in the oldest stars, born 10-15 billion years ago. These old and metal-deficient stars exhibit surprisingly large variations in concentrations of heavy elements. Understanding these variations promises to improve our understanding of the origin of the elements.

\section{General Nucleosynthesis}

The chemical composition of primordial solar system material is derived from analyses of the solar spectrum and carbonaceous chondrite meteorites ( 1 , and references therein). This elemental abundance pattern is used as a reference standard to compare with data from other stars, and we find it repeated so often among stars of the Galactic disk that is often considered as the cosmic or universal chemical composition. In this abundance distribution, $\mathrm{H}$ and $\mathrm{He}$ account for nearly all of the ordinary matter; the rest of the Periodic Table provides only trace elements. Among the remaining elements, the distribution features rapidly declining abundances with increasing atomic number $\mathrm{Z}$, an odd/even pattern with even- $\mathrm{Z}$ elements being more abundant than their immediate odd-Z neighbors, and relative abundance peaks that correspond to the more tightly bound atomic nuclei. 
$\mathrm{H}$ and $\mathrm{He}$ were created wholly or substantially in nuclear reactions that accompanied the Big Bang. Li also was made in the Big Bang and can sometimes be synthesized late in stellar lifetimes. The trio of $\mathrm{Li}, \mathrm{Be}$, and $\mathrm{B}$ are easily destroyed via proton-captures in the interiors of ordinary stars, but can be made in high-energy cosmic ray or neutrino spallation reactions on C, $\mathrm{N}$, and $\mathrm{O}$ target nuclei the interstellar medium (ISM). All other elements owe their existence to nuclear reactions in stellar interiors.

Charged-particle fusion reactions account for the abundances of nearly all of the isotopes of elements through the Fe-group $(6 \leq \mathrm{Z} \leq 30)$. These reactions are generally exothermic and thus ultimately are the energy sources that power the luminosities of the stars. The most common reactions are those that fuse four $\mathrm{H}$ nuclei into a He nucleus, and fuse three He nuclei into a $\mathrm{C}$ nucleus. Most stars such as the Sun are of relatively low mass and hence have interior temperatures and densities sufficient to create only the lighter elements. In the last stages and death throes of the rare, more massive stars $\left(\geq 8 \mathrm{M}_{\odot}\right)$ the interior temperatures and pressures are high enough to form Fe-group nuclei, but the fusion process is essentially the same as that which turns $\mathrm{H}$ into He.

This general model of nucleosynthesis does not explain how Periodic Table elements with $\mathrm{Z}>30$ are manufactured. It cannot be a common process, because the abundance of all these elements added together is $10^{8}$ times less than that of $\mathrm{H}$. Charged-particle fusion can make only relatively rare proton-rich isotopes of some of these elements, because nuclei of Fe-group elements are the most tightly bound of all, and the direct fusion of these nuclei is endothermic, requiring extra energy input to loosen the nuclear bonds (2). Instead, almost all isotopes of $Z>30$ elements are created from captures of free neutrons by Fe-peak target nuclei, hence these elements are collectively known as neutron-capture ( $n$-capture) elements. There are no electric charge barriers to resist $n$-capture reactions. Sources of free neutrons exist in interior fusion layers (3) during late quiescent evolutionary stages of stars over a wide mass range, 
and copious neutrons are liberated in the core regions during explosions of high-mass stars in Type II supernovae (SNe, 4). As nuclei accrete neutrons they become unstable and will undergo $\beta$-decay transformations of neutrons into protons, thereby moving to higher $\mathrm{Z}$ in the Periodic Table.

Neutrons may be liberated in He-fusion layers of stable stellar interiors via the reactions ${ }^{13} \mathrm{C}(\alpha, \mathrm{n}){ }^{16} \mathrm{O}$ and ${ }^{22} \mathrm{Ne}(\alpha, \mathrm{n}){ }^{25} \mathrm{Mg}$. These neutrons are then captured by heavy element seed nuclei. The neutron fluxes are sufficiently low $\left(\mathrm{N}_{n} \sim 10^{8} \mathrm{~cm}^{-3}\right)$ that nearly all possible $\beta$ decays will have time to occur between successive neutron captures. Synthesis of successively heavier isotopes will not stray from the so-called valley of $\beta$-stability (Fig. 1). This (relatively) slow chain of events is called the $s$-process, and it can account for about half of the isotopes of the $n$-capture elements.

Large numbers of free neutrons are created in high mass stars during the collapse of their cores to ultra-high densities as they commence their spectacular Type II SNe death throes. The reaction is simply $\mathrm{p}+\mathrm{e} \rightarrow \mathrm{n}+\nu$, and the neutron densities, $\mathrm{N}_{n}$, can reach $\gtrsim 10^{23} \mathrm{~cm}^{-3}$. In this situation extremely neutron-rich nuclei can be created in a matter of seconds as the rebound explosions rip the outer stellar layers from the condensed cores, without the necessity of having prior heavy element seed nuclei. The addition of free neutrons happens much more quickly than $\beta$-decay rates, and nuclei can be created as extreme as those at the neutron drip line (Fig. 1). The Type II SNe explosion happens so quickly that the source of free neutrons is quickly extinguished in the ejected material, and the extremely neutron-rich very heavy isotopes quickly $\beta$-decay back to the valley of $\beta$-stability. Creation of $n$-capture isotopes in this rapid manner is called the $r$-process.

Many $n$-capture isotopes can be reached exclusively by the $s$-process or the $r$-process paths, and some by both (Fig. 2). The composition of the Sun is a grand average of the nucleosynthesis of multiple generations of stars of a range of masses, and can only begin to be decoded through 
careful study of the meteoritic abundance data (5-8). The $s$-process contributions are readily estimated, because in this regime the abundances of individual isotopes are inversely proportional to their $n$-capture cross sections. ¿From comparisons of $s$-process predictions to the abundances of those isotopes which can only be synthesized in this manner, one can show that the whole distribution can be satisfactorily fit with just two types of $s$-process events that represent the outputs of many contributing stars over the history of the Galaxy. The first, so-called weak component, was responsible for the bulk of isotopes with atomic masses $A<90(Z<40, Z r)$; and the second, main component, produced the heavier isotopes.

The $r$-process contributions to meteoritic material are not easily understood, because isotopes formed in this way are synthesized on the very neutron-rich side of $\beta$-stability; such nuclei cannot be easily produced in the lab. Therefore, traditionally the $r$-process abundances have been inferred by first fitting s-process abundance distributions in the manner described above, then predicting the $s$-process fractions for isotopes that can be produced in both $r$ - and $s$-processes, then subtracting these from the total meteoritic abundances, and finally assigning the remainder to the $r$-process (Fig. 3) (9). The $r$ - and $s$-process solar-system abundance distributions show that not just individual isotopes but whole elements owe their synthesis predominantly to the $s$-process (e.g. Sr, Ba) or the $r$-process (e.g. Eu, Pt) in solar-system material.

\section{Neutron-capture Elements in the Early Galaxy}

The oldest stars of the Milky Way Galaxy, if they are not members of star clusters, can be identified in three ways: (i) residence in the Galactic halo, far from the disk; (ii) significantly different orbital motion than Sun, or (iii) low overall metallicity (10) of the Fe-group elements. For some stars all of these properties can be determined, and they turn out to be highly correlated. The metallicity identifier, relatively easy to assess from stellar spectra of modest resolution $(11,12)$, is based on the assumption that at a star's death, a fraction of the elements minted throughout 
its lifetime will be returned to the ISM, and subsequent star formation from this debris will produce stars with larger heavy element abundances. Therefore, to first order the older stars will be metal-deficient compared to the Sun. This principle can only be applied in the most general way, because it ignores all the details of the chemical and dynamical evolution of the Galaxy. Nevertheless, a star with Fe-peak metal content less than $1 \%$ of the Sun surely was formed at a time when the Galaxy was a small fraction of its present age, and its abundance set is the product of only a few prior element-donating stars.

We cannot study the $n$-capture elements of old low metallicity stars in the same detail as is possible for solar-system material, because individual isotopes generally cannot be detected in stellar spectra; only total elemental abundances can be deduced. Additionally, the atomic structures of many $n$-capture elemental species (e.g. the noble gases) render them spectroscopically inaccessible. Indeed, only a handful of $n$-capture elements have been analyzed in most metal-poor stars. Detailed abundance distributions are known for perhaps less than 20 of these stars. But this is one of the most active current areas of stellar spectroscopy, and work over the last two decades has shown that there is a huge amount of information about early Galactic chemical evolution encoded in the $n$-capture elements.

\section{Undeniable Scatter}

The most easily identified feature of $n$-capture elements in metal-poor stars is the very large starto-star variation in the bulk levels of these elements with respect to the lighter ones. For stars whose relative Fe-peak metallicities $(10)$ are less than a hundredth of the Sun's (i.e. $[\mathrm{Fe} / \mathrm{H}]<$ -2), $n$-capture element abundance levels in different stars range from at least $[n$-capture/Fe] -2 to +1 . This variation is probably an underestimate of the true range, and it is so large that simple visual inspection of stellar spectra demonstrate the effect beyond question $(7,13)$.

The star-to-star scatter increases dramatically with decreasing stellar metallicity (Fig. 4, 7). 
Europium is overwhelmingly synthesized by the $r$-process and is relatively easy to identify in the spectra of metal-poor Galactic halo stars. Thus, Eu provides a good indication of the $r$ process $n$-capture nucleosynthesis history of the Galaxy. Near $[\mathrm{Fe} / \mathrm{H}]=-3$ (which includes some of the oldest stars in the Galaxy, see $\S 3.3$ below), the $[\mathrm{Eu} / \mathrm{Fe}]$ ratio rises to peak of $\sim+1.7$ and varies from star-to-star by more than 2 dex (Fig. 4). The $[\mathrm{Eu} / \mathrm{H}]$ ratios in these stars are of course still less than that of the Sun, but the relative abundance of this $r$-process element to $\mathrm{Fe}$ in many of these stars can be much larger than the solar ratio. This star-to-star scatter diminishes with increasing metallicity tending toward younger stars.

While there could be several possible explanations for this scatter, the most straightforward interpretation suggests that the early Galaxy was chemically unmixed. The lifetime of a massive star is only about 10-30 My and Galactic mixing times are probably 100-300 My. Thus one would expect to produce pockets of chemically inhomogeneous ISM material. Over time, such disparities in the total abundance levels would be minimized by mixing throughout the Galaxy.

There is also a downward trend of $[\mathrm{Eu} / \mathrm{Fe}]$ ratios at higher metallicities (Fig. 4). Early in the history of the Galaxy Fe is produced from Type II SNe. These high-mass objects evolve rapidly and deposit Fe into the early Galaxy. The dominant Fe production in the Galaxy at later times and higher metallicities, however, comes from Type I SNe. Thus, Type I SNe with at least one lower-mass star progenitor have longer (than Type II SNe) evolutionary time scales, and could not have been present at the very earliest times in the history of the Galaxy. We see the increasing influence of this Galactic Fe production, with decreasing values of [Eu/Fe], occurring near $[\mathrm{Fe} / \mathrm{H}] \sim-1$, which might take on the order of a billion years after the Galaxy formed. 


\section{Dominance of the $r$-Process}

One of the most well-studied Galactic halo stars is the ultra-metal poor (UMP) giant star CS 22892-052 $([\mathrm{Fe} / \mathrm{H}]=-3.1)(14,15,16)$. In fact, ground-based, and now space-based, observations have probably detected more elements in CS 22892-052 than any other star except the Sun! A primary reason that CS 22892-052 has been so extensively observed is that it has very large overabundances, with respect to $\mathrm{Fe}$, of the $n$-capture elements (e.g., $[\mathrm{Eu} / \mathrm{Fe}] \simeq+1.7)$. The $n$-capture element abundance determinations for this star are similar to the solar system $r$-process abundance curve (Fig. 5). Although the total abundance levels of these heavier $n$ capture elements are below the solar levels, the $n$-capture element abundances in this star are the same as the solar system elemental $r$-process-only abundances. This similarity in abundance levels has now been observed in several other halo stars (17). There is an additional, apparently rare, anomalous abundance class of UMP stars: those that are $\mathrm{C}$-rich with a large range in $\mathrm{Fe}$ peak and $n$-capture abundance distributions $(18,19)$. These stars may offer other insights into the conditions at the very earliest times in the Galaxy, but will not be considered further here.

These halo star abundance signatures provide insight into the conditions in the early Galaxy and the types of stars that synthesize the $n$-capture elements. For example, $\mathrm{Ba}$ is overwhelmingly produced in the $s$-process, with a small contribution from the $r$-process, in solar system material. The abundance comparisons for the metal-poor but neutron-rich halo stars such as CS 22892-052, however, seem to indicate that early in the history of the Galaxy, all of the elements, even those like Ba now thought to be associated with the $s$-process, were synthesized instead in the $r$-process. The presence of these $r$-process abundances in these metal-poor and old stars also suggests that whatever the astrophysical site for the $r$-process, it was rapidly evolving. This follows because the time delay between the formation of the Galaxy and the old halo stars was likely millions, not billions, of years. Thus, the first stars, the progenitors of the halo stars, had to have evolved quickly, synthesized the $r$-process elements and ejected them 
into the ISM before the formation of the currently observed UMP stars. We note that the main component of the $s$-process is identified with low- or intermediate-mass stars (i.e., all stars with masses less than $8 \mathrm{M}_{\odot}$ ) with fairly long evolutionary time scales (20). These stars, therefore, would not have had time to have synthesized the first elements in the Galaxy.

The abundance comparisons for CS 22892-052 (Fig. 5), along with similar results for several other metal-poor halo stars suggests that the $r$-process for the heavier $n$-capture elements is robust. In other words, we see the same relative proportions of these elements in stars that are many billions of years different in age, suggesting that this process has operated in a fairly consistent manner over large periods of time in the history of the Galaxy. This result may ultimately help to constrain the conditions that occur in the $r$-process, and hopefully help to determine how and where in nature these nuclei and elements are synthesized.

However there could be more than one type of $r$-process occurring in multiple sites. This is suggested by the lack of agreement between some of the lighter $n$-capture element abundances and the same scaled solar $r$-process curve that is consistent with the heavier such elements (Fig. 5). The abundances of several elements in the range of $40 \leq \mathrm{Z} \leq 50$ for at least three stars fall below the Solar curve $(16,21,22)$, suggesting that there are two $r$-process sites. Possibilities for these sites could be different mass ranges or frequencies of SNe or perhaps a combination of SNe and neutron-star binaries (17), although some neutron-star binaries might have longer evolutionary timescales than typical SNe. Alternatively, two entirely different sets of conditions in the same site (perhaps a $\mathrm{SNe}$ ) could produce the entire range of $n$-capture abundances in these halo stars.

Previous work (23) has employed $\mathrm{Ba} / \mathrm{Eu}$ ratios to assess $s$-/r-process ratios in large stellar samples. Such surveys have shown that at low metallicities are generally consistent with the solar $r$-process ratio (17). However, there is an unsettlingly large amount of scatter in these $\mathrm{Ba} / \mathrm{Eu}$ data at any metallicity. It has not been clear whether this is an intrinsic scatter or the result 
of observational uncertainty. The probable observational culprit is $\mathrm{Ba}$ : with a large number of isotopes (each with a different sensitivity to the $r$ - and $s$-process) and with generally only very strong lines available for analysis, abundance analyses of Ba have large uncertainties. These difficulties have led to searches for other elements that might be employed for these chemical evolution comparisons.

Lanthanum, which is also mostly an $s$-process element in solar system material and has only one stable isotope, may be more useful for assessing $r$-process versus $s$-process contributions. Preliminary results from a study on the variation in halo star $\mathrm{La} / \mathrm{Eu}$ ratios as a function of metallicity (24) indicate that $\mathrm{La} / \mathrm{Eu}$ values are consistent with the solar $r$-process value at lowest metallicities, and the star-to-star scatter is fairly small. At the earliest Galactic times the bulk of the heavier elements were synthesized in the $r$-process. There is a general rise in $\mathrm{La} / \mathrm{Eu}$ ratios near a metallicity of $[\mathrm{Fe} / \mathrm{H}] \simeq-2$, because of an increased production of the $s$-process element La from lower mass stars $\left(\mathrm{M} \leq 5 \mathrm{M}_{\odot}\right)$, which begin to contribute their ejecta to the ISM. These low- to intermediate-mass stars take billions of years to evolve before they are able to inject large amounts of $s$-process rich material into the Galaxy. While the major onset of this Galactic nucleosynthesis may occur near $[\mathrm{Fe} / \mathrm{H}]=-2$, there is a range in metallicity over which there is evidence of some Galactic s-processing (7).

\section{The Age of the Galaxy}

The abundance data for certain radioactive $n$-capture elements can be employed to determine the ages of the oldest individual halo stars, and in this manner to date the age of the Galaxy. These radioactive-age determinations, first suggested in (25), rely upon the known decay time (i.e., half life) of long-lived radioactive nuclei, frequently referred to as chronometer nuclei. Thorium, for example, has a half-life of 14 Gy. Large telescopes and efficient high-resolution spectrographs have allowed stellar Th detections and the related age estimates to be made. 
Recent studies have found a range of age estimates of approximately 11-15 Ga for halo UMP stars (17). Thorium has also been detected in some giant stars in the globular cluster M15 and has been used to determine a radioactive age estimate of $14 \pm 4 \mathrm{Ga}(26)$.

Recently U was detected in the spectrum of the UMP star CS 31082-001 (27). Abundance comparisons of this element to Th and to several other elements led to an age estimate for this star of $12.5 \pm 3 \mathrm{Ga}$, in concordance with other age estimates based on the Th chronometer. Abundance comparisons between Th and $\mathrm{U}$, near neighbors in mass number, might allow for more reliable age determinations than comparisons among elements widely separated in atomic mass. In this regard we note the divergent chronometric age estimates (28) obtained for CS 31082-001 based upon Th/U and Th/Eu. The Th/Eu value is so unrealistically low that there may be uncertainties in employing only $\mathrm{Th} / \mathrm{Eu}$ for age estimates, due to varying $r$-process element production at the higher mass end of the distribution. On the other hand another tentative $\mathrm{U}$ detection has been recently reported in a metal-poor halo star (21), and in this case, $\mathrm{Th} / \mathrm{U}$ and $\mathrm{Th} / \mathrm{Eu}$ give comparable age values. At this point, therefore, it is not clear if CS 31082-001, with its very large overabundances of $\mathrm{Th}$ and $\mathrm{U}$ is somehow unusual, but the results for that star (at least) do suggest that $\mathrm{Th} / \mathrm{U}$ is a more reliable chronometer than $\mathrm{Th} / \mathrm{Eu}$.

A critical factor in Th and $U$ age estimates is estimating the initial abundances before decay of these elements generated in an $r$-process site. Prediction of initial abundances requires knowledge of both the physical conditions of the site and the nuclear properties (e.g., masses) of the neutron-rich nuclei. With little experimental data available, the nuclear properties must be obtained from theoretical models. Advances in all of these areas will strengthen the radioactive dating technique.

The age of the Galaxy has also been determined using the known evolutionary times for low-mass stars leaving the main sequence (i.e., the "turn off point") of globular clusters. Such age estimates are between 12-15 Ga (29). Recent cosmological studies from observations of 
(Type Ia) SNe indicate that the Universe is about $15 \mathrm{Ga}(30,31)$. The delay between the Big Bang and the beginning of galaxy formation is not well determined, but typical time scales of about $1 \mathrm{Ga}$ have been commonly assumed. Radioactive age determinations are consistent with these globular cluster and cosmological age estimates. Nevertheless, radioactive dating is a relatively unexploited technique and the age estimates still suffer from some uncertainties. These uncertainties could be reduced and improvements made with additional data, particularly of simultaneous Th and $\mathrm{U}$ abundances in additional stars.

\section{The Earliest Stellar Generations in the Galaxy}

Most of the well-studied low-metallicity halo stars have relatively large abundances of $n$-capture elements that are detectable in their stellar spectra. Much less is known about halo stars with lower, or undetectable, abundance levels of these elements. Likewise, there are still few highresolution studies of UMP $([\mathrm{Fe} / \mathrm{H}]<-3)$ stars. Thus, we have little knowledge of the abundance patterns of the $n$-capture elements in these early stars. We do have some recent clues that might suggest a complex nucleosynthesis history at the earliest times in the history of the Galaxy. One such piece of evidence is the large difference in the abundance ratios of $\mathrm{Sr} / \mathrm{Ba}$ in groups of stars with high and low total amounts of $n$-capture elements. The $r$-process-rich stars have low $\mathrm{Sr} / \mathrm{Ba}$ ratios that are mostly consistent with the solar $r$-process ratio. The $r$-process-poor stars, on the other hand, have in some cases large values of $\mathrm{Sr} / \mathrm{Ba}$ (17). The significance of large $\mathrm{Sr} / \mathrm{Ba}$ ratios in some of these stars might be their low values of $\mathrm{Ba}$, and by inference, of other heavier $n$-capture elements. These observational comparisons suggest that some of the progenitors of the halo stars were able to synthesize lighter $n$-capture elements, such as $\mathrm{Sr}$, but produced very little of the heavier n-capture elements like Ba.

A related clue about the early chemical history of the Galaxy is provided by elementby-element comparisons between stars with large overabundances of $r$-process elements (e.g, 
CS 22892-052) with those that are deficient in these elements (e.g., HD 122563). The levels of the heavier $n$-capture elements in $r$-process-rich stars like CS 22892-052 have similar proportions as the solar system $r$-process abundances. The situation is different for, at least, the $r$-process poor star HD 122563, where the abundance data seem to depend upon, and fall with, increasing $\mathrm{Z}$ (17). This might suggest an incomplete $r$-process in the halo progenitors or that stars like HD 122563 formed so early in the Galaxy that the main sites for the r-process (e.g., SNe from a certain progenitor mass range) had not yet formed. These possibilities might also explain the large $\mathrm{Sr} / \mathrm{Ba}$ abundance ratio observed in some of the $r$-process poor stars, and are also supported by recent observations of UMP stars (7). The observations in those few stars, although lacking extensive heavier element data, indicate large overabundances in $\mathrm{C}, \mathrm{N}$ or $\mathrm{O}$ but very low or undetectable levels of heavier elements like $\mathrm{Ba}$ and $\mathrm{Eu}(32,33)$. Thus, these $\mathrm{C}$-rich stars might be exhibiting ejecta from some of the earliest Galactic stellar generations before the main onset of the $r$-process.

We also do not know a great deal about the earliest Galactic stellar progenitors - these stars have long since disappeared. Recent theoretical studies (e.g., 34) have suggested that this first generation was composed of very massive $\left(>100 \mathrm{M}_{\odot}\right)$ objects, which could have synthesized some small amounts of heavier elements and perhaps Fe. No stars have ever been detected with zero metals, containing only the products of the big bang, $\mathrm{H}$ and He. Because some UMP stars, e.g., CS 22892-052, show "complete" r-process signatures, some early progenitor stars, in existence when $-4<[\mathrm{Fe} / \mathrm{H}]<-3$, were already capable of synthesizing the entire range of $n$-capture elements, and not just the lighter elements.

\section{Future Directions}

Despite much progress there are still a number of unanswered questions and poorly understood areas of study in nuclear astrophysics, and the early history, chemical evolution and age of the 
Galaxy. One important problem to solve is the astrophysical site, or sites, for the $r$-process. Ever since its initial naming in $1957(36,37)$ it has been suspected that this process occurred somewhere in exploding SNe. Nevertheless, decades of study have still not been able to unambiguously identify the stellar or SNe environment (or even the basic conditions) in which the $r$-process might occur. Understanding the very complicated physics in a SNe and successfully modeling an explosion could also help greatly in solving this problem.

There also still remain great uncertainties in the $r$-process related to the basic nuclear physics involving these unstable, very neutron-rich nuclei. Experimental nuclear data are slowly becoming available, along with increasingly more precise theoretical prescriptions of the properties of these nuclei.

Abundance observations in the very metal-poor and UMP stars have suggested that the lighter $(\mathrm{Z}<56)$ and heavier $n$-capture elements might be synthesized in different $r$-process environments. However more stellar abundance data, particularly for $40 \leq \mathrm{Z} \leq 50$, are needed to establish, whether there are multiple $r$-process sites. Another area of future research that will prove fruitful is the early chemical history of the Galaxy. Are there, for example, other stars like HD 122563, that show low levels of heavy elements? Does that point to a type of incomplete $r$-process in some early progenitors or was there a time delay before the major $r$ process sites could evolve? Why was the production of the lighter $n$-capture elements favored over the heavier such elements in some stars? At what metallicity and/or time did the the first main $r$-process sites come into existence and when was the first Fe synthesized? All of these issues will require extensive new observational and theoretical studies. 


\section{References and Notes}

1. N. Grevesse and A. J. Sauval, 1998, Space Sci. Rev., 85, 161

2. The high temperatures that would be required (to overcome the electric Coulomb barriers) for fusion of nuclei beyond iron would also result in a large number of high energy photons. These photons in turn result in photo-disintegration of nuclei that suppress any possible chargeparticle fusion reactions.

3. $\mathrm{H}$ fusion in stars occurs in the inner core (approximately the innermost $10 \%$ of the star by mass) and lasts for about $90 \%$ of the star's total life. After the exhaustion of $\mathrm{H}$ in the core the star will then develop a thin (thousands of $\mathrm{km}$ ) $\mathrm{H}$ fusion shell outside of the inert He core. Later when the temperature rises to above 100 million degrees the He core will fuse $\mathrm{C}$ and $\mathrm{O}$. When the $\mathrm{He}$ is finally depleted in the core, a thin He fusion shell, outside of the now $\mathrm{C} / \mathrm{O}$ core but interior to the $\mathrm{H}$ fusion shell, will ignite. These later fusion stages occur only during the last approximately $10 \%$ of a star's life.

4. Supernovae ( $\mathrm{SNe}$ ) are observationally categorized by the presence (Type II) or absence (Type I) of hydrogen spectral lines. Further, Type II SNe are normally thought to result from the collapse and explosion of single massive, short-lived stars. Type I SNe are thought to be phenomena of lower-mass, longer-lived binary star systems, with the eventual explosion and complete destruction of the white dwarf member of the binary.

5. A. G. W. Cameron, 1982, in Essays in Nuclear Astrophysics, eds. C. A. Barnes, D. D. Clayton, and D. N. Schramm (Cambridge Univ. Press, Cambridge) p. 23.

6. F. Käppeler, H. Beer, and K. Wisshak, 1989, Rep. Prog. Phys. 52, 945

7. D. L. Burris, C. A. Pilachowski, T. A. Armandroff, C. Sneden, J. J. Cowan, and H. Roe, 2000, Astrophys. J., 544, 302

8. C. Arlandini, F. Käppeler, K. Wisshak, R. Gallino, M. Lugaro, M. Busso, and O. Straniero, 1999, Astron. Astrophys., 525, 886 
9. Caution is recommended in interpreting the $r$-process fractions of isotopes with extreme dominance by the $s$-process: a small error $(\sim 10 \%)$ in the $s$-process fraction in these cases leads to a large uncertainty $((\geq 25 \%)$ in the remainder $r$-process fraction.

10. Metallicity is usually defined to be the Fe content of a star. Stellar spectroscopists employ two logarithmic abundance notations. The first is an absolute abundance for element A normalized to the $\mathrm{H}$ abundance: $\log \epsilon(\mathrm{A}) \equiv \log _{10}\left(\mathrm{~N}_{\mathrm{A}} / \mathrm{N}_{\mathrm{H}}\right)+12.0$. With this definition $\log \epsilon(\mathrm{H})=$ 12. The second is an abundance relative to the solar system for elements $A$ and $B:[A / B] \equiv$ $\log \left\{\left(\mathrm{N}_{A} / \mathrm{N}_{B}\right)_{\text {star }} /\left(\mathrm{N}_{A} / \mathrm{N}_{B}\right)_{\text {Sun }}\right\}$. With this definition $[\mathrm{Fe} / \mathrm{H}]$ is normally used as a surrogate for overall metallicity, and for example $[\mathrm{Eu} / \mathrm{Fe}]$ tells the difference in stellar and solar $\mathrm{Eu} / \mathrm{Fe}$ abundance ratios.

11. H. E. Bond, 1980, Astrophys. J. Suppl. Ser., 44, 517

12. T. C. Beers, G. W. Preston, and S. A. Shectman, 1992, Astron. J., 103, 1987

13. A. McWilliam, G. W. Preston, C. Sneden, and L. Searle, 1995, Astron. J., 109, 2757

14. C. Sneden, et al. 1996, Astrophys. J., 467, 819

15. C. Sneden, et al. 2000, Astrophys. J., 533, L139

16. C. Sneden, et al. 2002, in preparation

17. J. W. Truran, J. J. Cowan, C. Sneden and C. Pilachowski, 2002, Publ. Astron. Soc. Pac., submitted

18. W. Aoki, J. E. Norris, S. G. Ryan, T. C. Beers, and H. Ando, 2002, Astrophys. J., 576, 141.

19. W. Aoki, J. E. Norris, S. G. Ryan, T. C. Beers, and H. Ando, 2000, Astrophys. J., 536, 97.

20. M. Busso, R. Gallino, and G. J. Wasserburg, 1999, Annu. Rev. Astron. Astrophys., 37, 239

21. J. J. Cowan, et al. 2002, Astrophys. J., 572, 861 
22. V. Hill et al., 2002, Astron. Astrophys., 387, 560

23. M. Spite, and F. Spite 1978, Astron. Astrophys., 67, 23

24. J. Simmerer, et al. 2002, in preparation

25. H. R. Butcher, 1987, Nature, 328, 127

26. C. Sneden, et al., 2000, Astrophys. J., 536, L85

27. R. Cayrel, et al. 2001, Nature, 409, 691

28. H. Schatz, et al. 2002, Astrophys. J., in press

29. F. Pont, F., M. Mayor, C. Turon, and D. A. Vandenberg, 1998, Astron. Astrophys., 329, 87

30. A. G. Riess, et al. 1998, Astron. J., 116, 1009

31. S. Perlmutter, et al. 1999, Astrophys. J., 517, 565

32. J. E. Norris, S. G. Ryan, and T. C. Beers, 1997, 2001, Astrophys. J., 561, 1034

33. E. Depagne, et al. 2002, Astron. Astrophys., 390, 187

34. Y.-Z. Qian, and G. J. Wasserburg, 2001, Astrophys. J., 559, 925

35. We thank all of our colleagues for helpful conversations and insightful comments. We are grateful to Peter Möller for providing us with a copy of Figure 1. Partial support for this research was provided by the National Science Foundation (AST-9987162 to CS and AST9986974 to JJC) and by the Space Telescope Science grant GO-08342. JJC thanks the University of Texas at Austin Department of Astronomy John W. Cox Fund for partial support while this paper was being written.

36. E. M. Burbidge, G. R. Burbidge, W. A. Fowler, and F. Hoyle, 1957 Rev. Mod. Phys., 29, 547

37. A. G. W. Cameron, 1957, Chalk River Report CRL-41

38. P. Möller, Nix, \& K.-L. Kratz, 1997, Atomic Data Nuclear Data Tables, 66, 131 


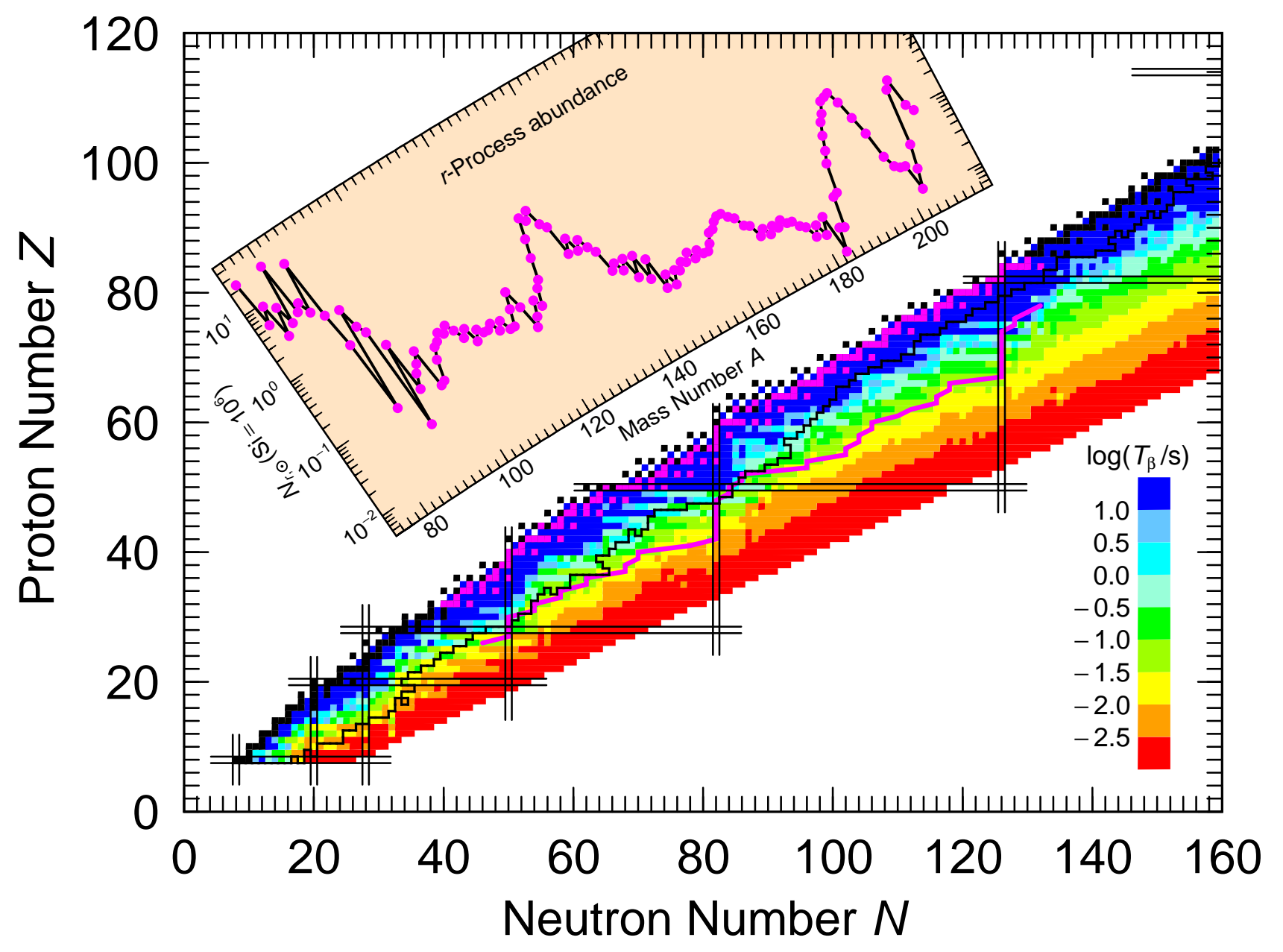

Figure 1: A chart of the nuclides (after 38). At a given proton (atomic) number, isotopes toward the left are proton-rich and those to the right are th neutron-rich ones that are the subject of this paper. The stable nuclides are marked by black boxes and $n$-capture in s-process synthesis occurs near these nuclei close to the "valley of beta stability." The jagged diagonal black line represents the limit of experimentally determined properties of nuclei and the magenta line the r-process "path." Vertical and horizontal black lines represent closed neutron or proton shells, sometimes referred to as "magic numbers." Color shading denotes the different (log) timsecales for beta-decay. 


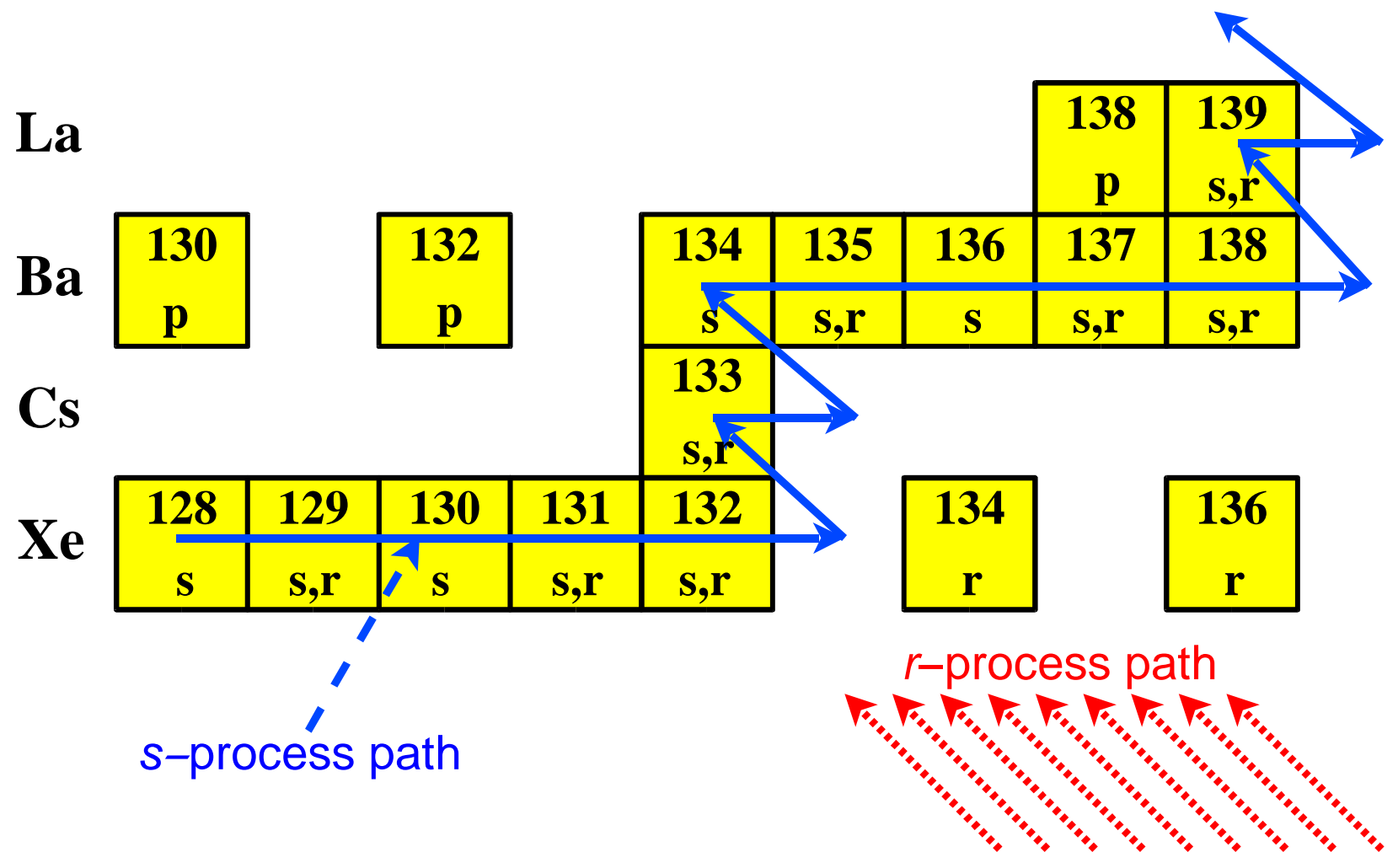

Figure 2: A small portion of the chart of the nuclides, illustrating isotopes built by $r$ - and $s$ process syntheses. Only the stable isotopes are shown in this figure. In each box the number is the atomic mass of the isotope, and the letter(s) give the nucleosynthesis process(s) that can contribute to the growth of the isotope. 


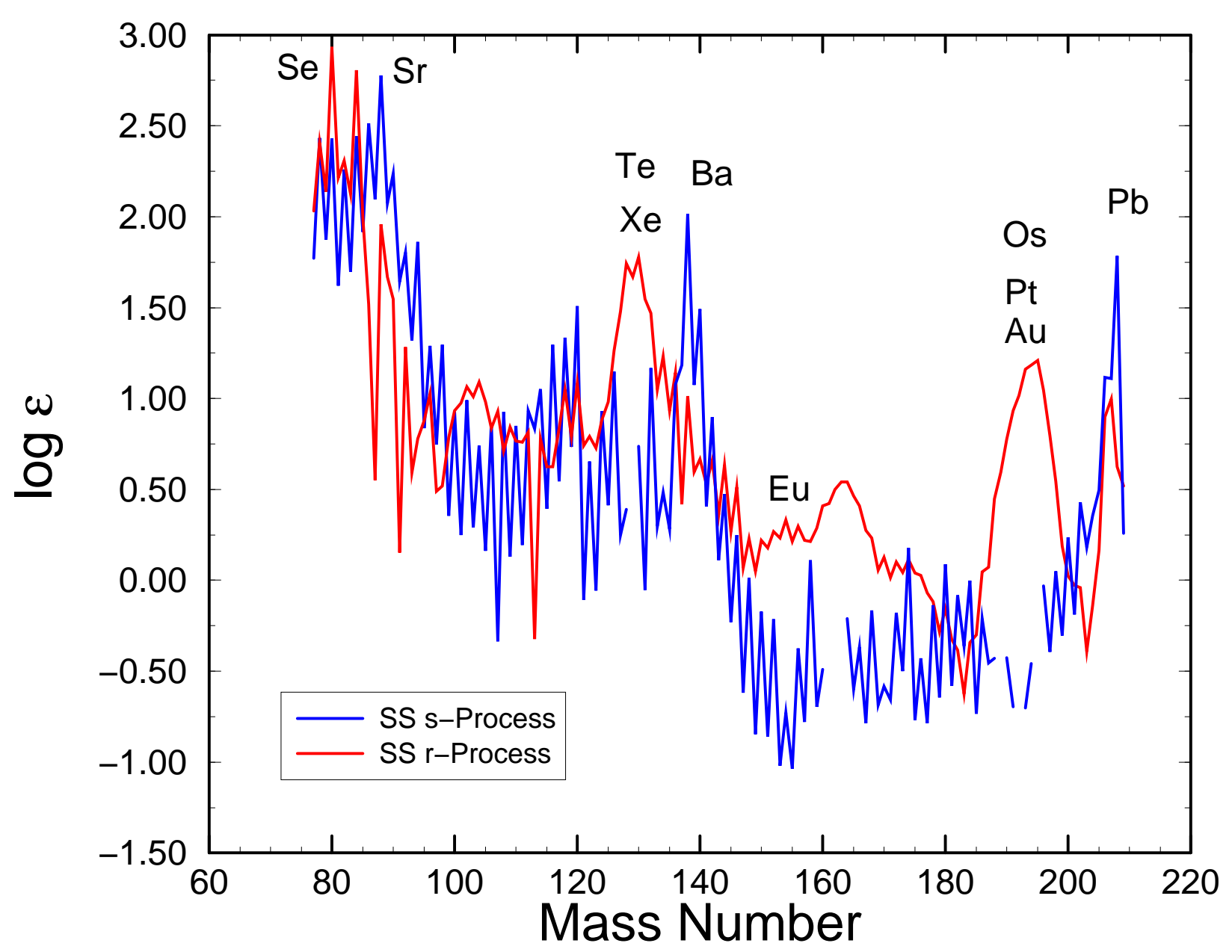

Figure 3: The breakdown of solar system (meteoritic) $n$-capture isotopic abundances into $r$ - and $s$-process components (based upon 6 ). The approximate mass numbers corresponding to some prominent elements are noted. 


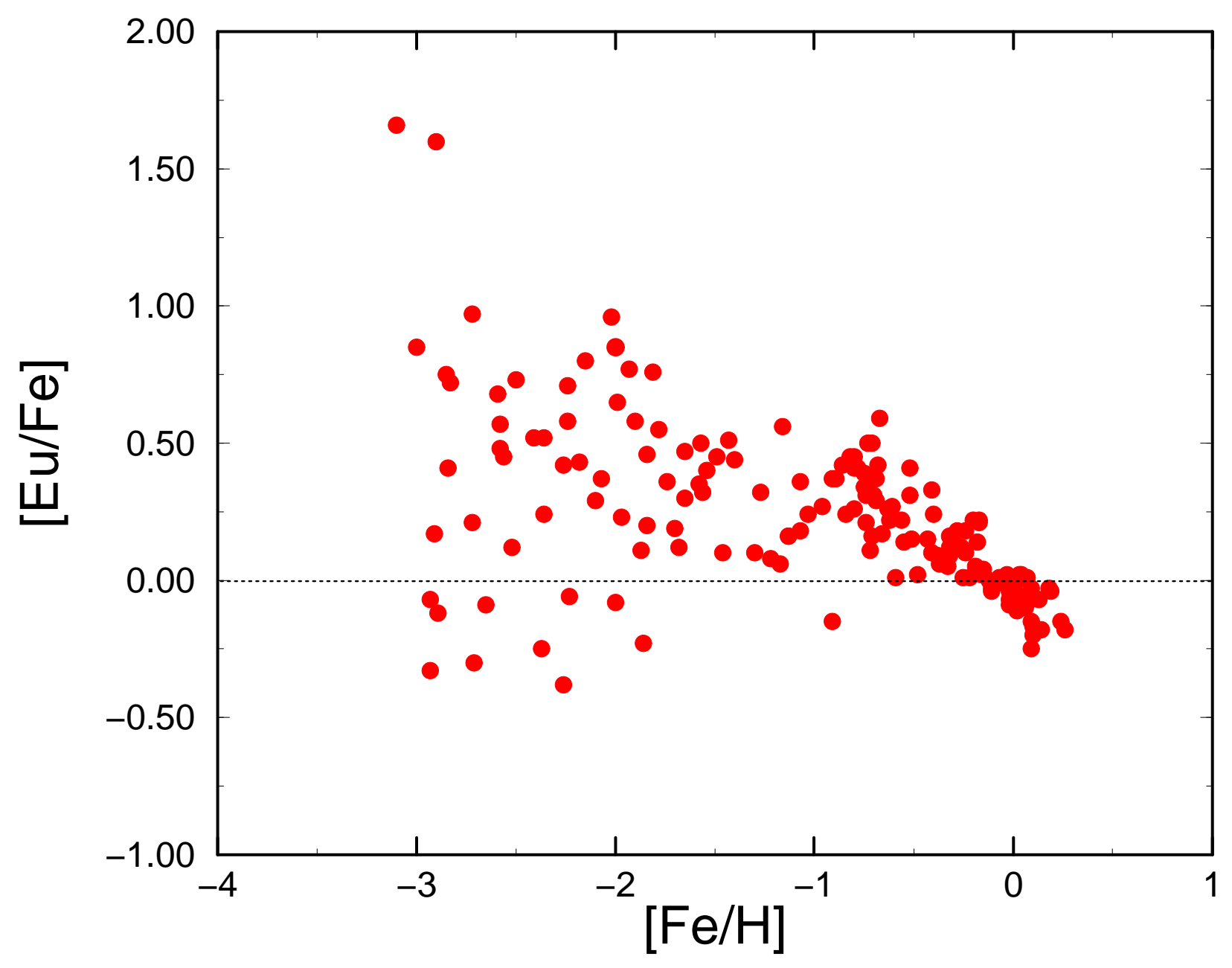

Figure 4: The ratio $[\mathrm{Eu} / \mathrm{Fe}]$ is displayed as a function of $[\mathrm{Fe} / \mathrm{H}]$ from various surveys of halo and disk stars as detailed in (17). The dotted line represents the solar value. 


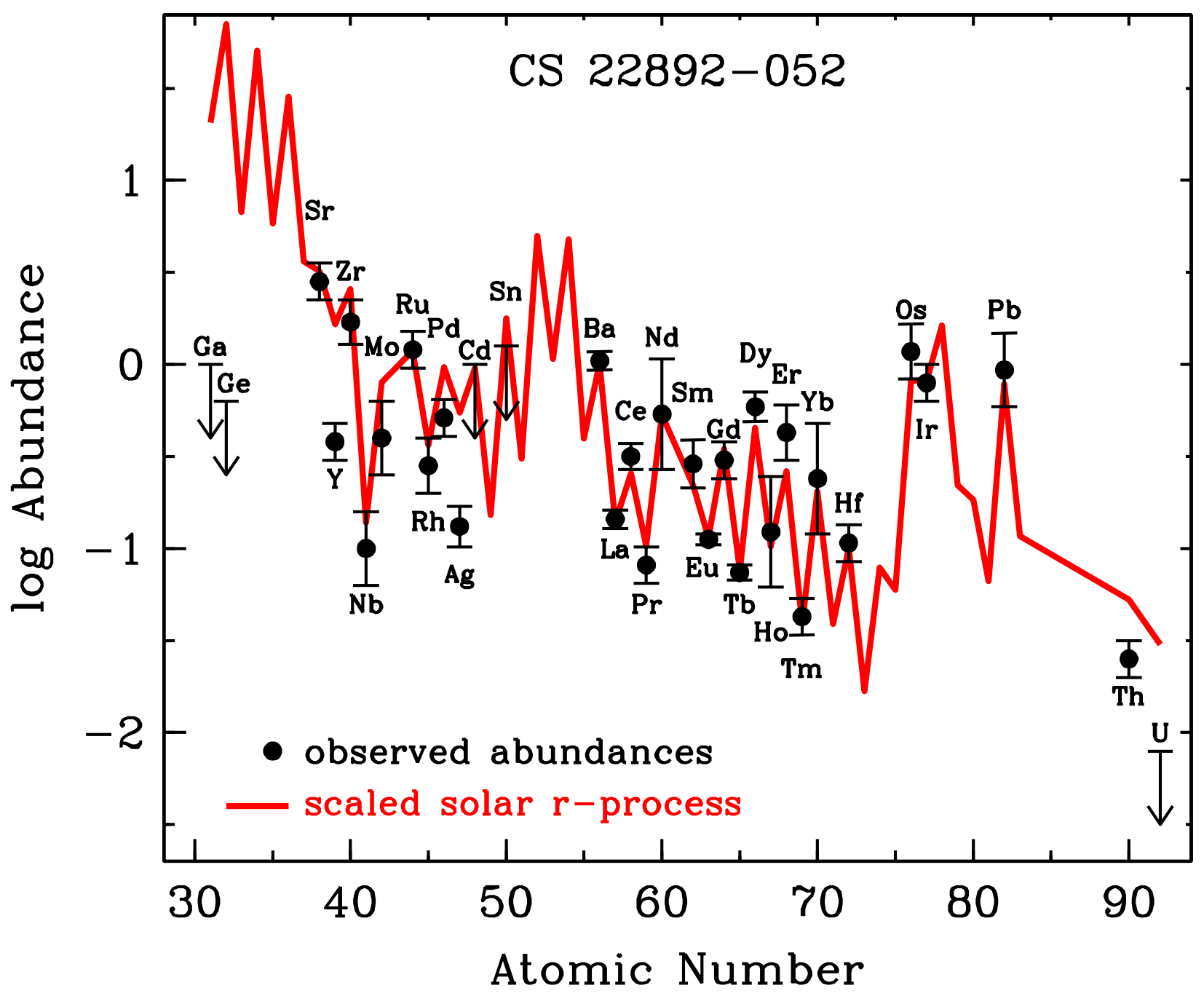

Figure 5: Abundance comparison of elements in the ultra-metal-poor halo star CS 22892-052 (points with error bars) and a scaled solar system $r$-process abundance curve (16). The dashed line is based upon nuclear physics (i.e., neutron capture cross section) experiments ( 6 ) to determine the solar system $s$ - and $r$-process isotopic (see Figure 3), and hence, elemental abundance determinations. 


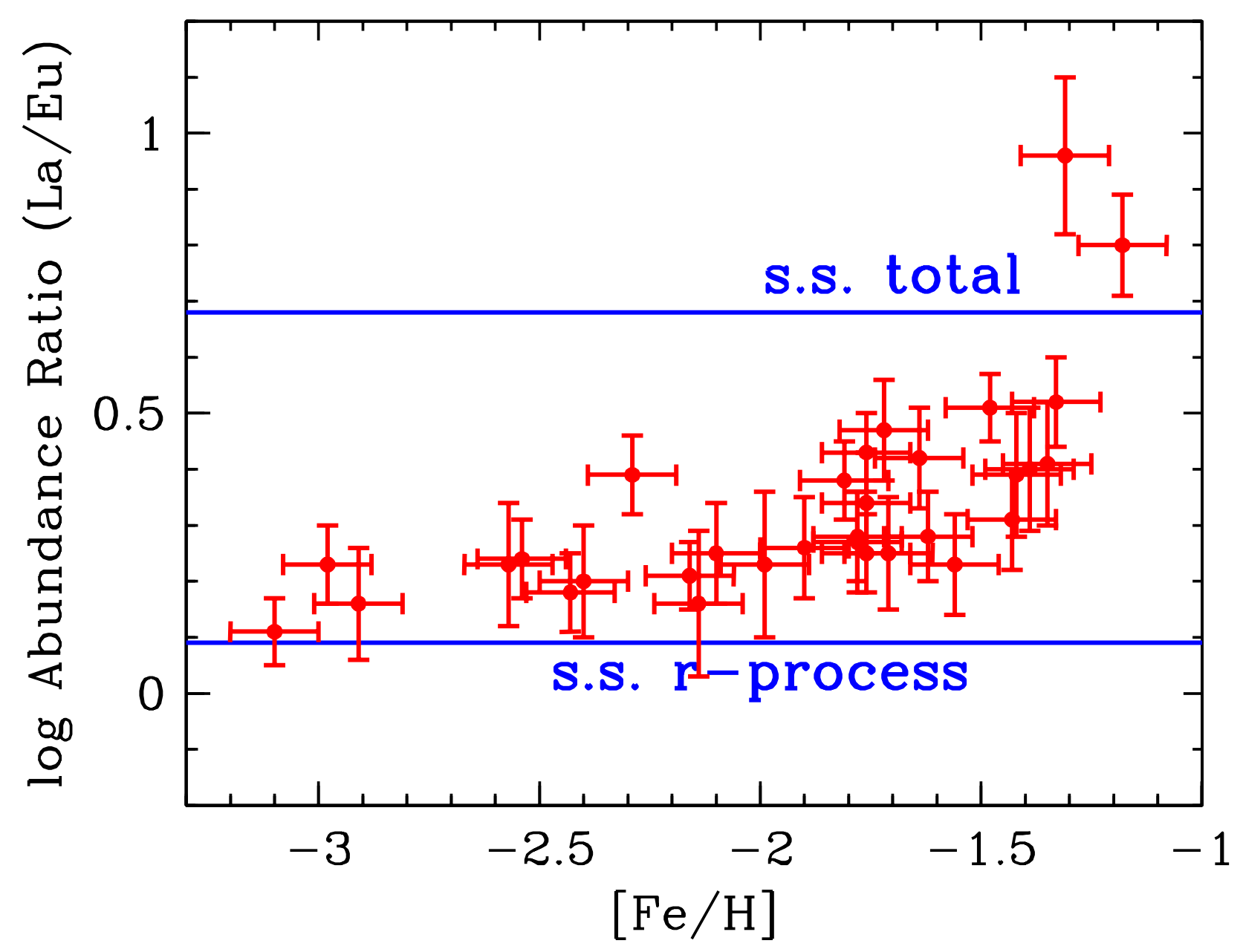

Figure 6: La/Eu ratios for a variety of metal-poor stars as a function of metallicity (24). The horizonal lines denote the total and r-process only solar system abundance ratios. 